OPEN ACCESS

Edited by: Ilhem Messaoudi, University of California, Irvine, United States

Reviewed by: Michael Z. Zulu, University of California, Irvine, United States J. Zachary Porterfield, University of Kentucky, United States

${ }^{*}$ Correspondence:

Mkunde Chachage chachage.mkunde@udsm.ac.tz Wilbert Mbuya wmbuya@nimr-mmrc.org

Specialty section: This article was submitted to Virus and Host, a section of the journal Frontiers in Cellular and Infection Microbiology

Received: 07 June 2021 Accepted: 13 October 2021 Published: 09 November 2021

Citation:

Mbuya W, Mwakyula I, Olomi W, Agrea $P$, Nicoli $F$, Ngatunga $C$, Mujwahuzi L, Mwanyika $P$ and

Chachage M (2021) Altered Lipid Profiles and Vaccine Induced-Humoral Responses in Children Living With HIV on Antiretroviral Therapy in Tanzania. Front. Cell. Infect. Microbiol. 11:721747. doi: 10.3389/fcimb.2021.721747

\section{Altered Lipid Profiles and Vaccine Induced-Humoral Responses in Children Living With HIV on Antiretroviral Therapy in Tanzania}

\author{
Wilbert Mbuya ${ }^{1 *}$, Issakwisa Mwakyula ${ }^{2}$, Willyelimina Olomi ${ }^{1}$, Peter Agrea ${ }^{1}$, \\ Francesco Nicoli ${ }^{3}$, Cecilia Ngatunga ${ }^{4}$, Leodegard Mujwahuzi ${ }^{2}$, \\ Paul Mwanyika ${ }^{5}$ and Mkunde Chachage ${ }^{1,6 *}$
}

${ }^{1}$ National Institute for Medical Research (NIMR), Mbeya Medical Research Centre (MMRC), Mbeya, Tanzania, ${ }^{2}$ Department of Internal Medicine, Mbeya Zonal Referral Hospital and University of Dar es Salaam Mbeya College of Health and Allied Sciences, Mbeya, Tanzania, ${ }^{3}$ Department of Chemical, Pharmaceutical and Agricultural Sciences, University of Ferrara, Ferrara, Italy, ${ }^{4}$ Department of Radiology, Mbeya Zonal Referral Hospital and University of Dar es Salaam Mbeya College of Health and Allied Sciences, Mbeya, Tanzania, ${ }^{5}$ Department of Paediatric, Mbeya Zonal Referral Hospital and University of Dar es Salaam Mbeya College of Health and Allied Sciences, Mbeya, Tanzania, ${ }^{6}$ Department of Microbiology and Immunology, University of Dar es Salaam - Mbeya College of Health and Allied Sciences (UDSM-MCHAS), Mbeya, Tanzania

People living with HIV, even under therapy, have a high burden of age-related co-morbidities including an increased risk of dyslipidemia (which often predisposes to cardiovascular diseases) and immune-aging. In this study, lipid profiles and antibody responses to measles and pertussis toxin vaccines were compared between ART experienced HIV+ children ( $n=64)$ aged 5-10 years, and their age- and sex-matched HIV- controls $(n=47)$. Prevalence of high-density lipoprotein cholesterol (HDL-c) and triglyceride-driven dyslipidemia was higher among treated $\mathrm{HIV}+$ children than in controls (51.6\% vs $27.7 \%$ respectively, $p<0.019)$. In a multivariate Poisson regression model adjusted for age, sex and BMI, the association between low HDL-c, hypertriglyceridemia and HIV remained significantly high (for HDL-C: ARR: 0.89, 95\% Cl: $0.82-0.96$, $\mathrm{p}=0.003$; for triglycerides: ARR: $1.54,95 \% \mathrm{Cl}: 1.31-1.81, \mathrm{p}<0.001)$. Among HIV+ children, the use of lopinavir/ritonavir, a protease-based antiretroviral therapy was also associated elevation of triglyceride levels $(p=0.032)$. Also, HIV+ children had a 2.8-fold reduction of anti-measles IgG titers and 17.1-fold reduction of anti-pertussis toxin IgG levels when compared to HIV- children. Our findings suggest that dyslipidemia and inadequate vaccine-induced antibody responses observed in this population of young African HIV+ children might increase their risk for premature onset of cardiovascular illnesses and acquisition of preventable diseases.

Keywords: HIV, children, dyslipidemia, cardiovascular disease, immune senescence, childhood vaccines, ART 


\section{INTRODUCTION}

Despite the importance of ART in reducing HIV morbidity and mortality, people living with HIV (PLWH) have, even under therapy, increased morbidity and decreased life expectancy (Antiretroviral Therapy Cohort Collaboration et al., 2009; Modrich et al., 2010; IeDEA Pediatric Working Group, 2017). Furthermore, compared to the general population, PLWH have an accelerated aging process, which is often associated with a high burden of age-related co-morbidities (Deeks and Phillips, 2009; Guaraldi et al., 2011).

There is a growing body of literature that recognizes an increased risk of age-related conditions such as cardiovascular diseases (CVD) for HIV positive persons at a younger age compared to that of their age-matched uninfected counterparts (Maggi et al., 2017). Several studies have documented a higher prevalence of traditional CVD risk factors such as obesity, diabetes, smoking and dyslipidemia in PLWH (Rahmanian et al., 2011; Tien et al., 2012; Paisible et al., 2015). HIVassociated dyslipidemia is often characterized by elevated levels of triglycerides and/or heightened low-density lipoprotein cholesterol (LDL-c) levels and reduced high-density lipoprotein cholesterol (HDL-c). Certain types of ART also contribute to a high prevalence of dyslipidemia in PLWH (Nduka et al., 2015), further contributing to increased risk of CVD. Apart from dyslipidemia and other traditional CVD risk factors, long-term ART exposure and HIV-induced chronic inflammation and immune activation that persists despite the use of ART may also drive cellular aging and thus contribute to increasing the risk for CVD in HIV+ people (McDonald and Kaltman, 2009; Syed et al., 2013; Maggi et al., 2017).

Atherosclerosis begins in childhood, prospectively increasing the risk of developing CVD (Daniels et al., 2011; Jaquith et al., 2013), indicating the importance of characterizing the impact of HIV and ART on CVD already at an early age. Indeed, HIV+ children are projected to display mortality rates 30 times higher than uninfected children due to age-related disorders (Brady et al., 2010; Guaraldi et al., 2011; Jaquith et al., 2013; Chiappini et al., 2014; Guaraldi and Palella, 2017). Therefore, it is plausible to think that the accelerated aging process described in adults affects children living with HIV as well. Currently, 1.8 million children live with HIV infection worldwide, with the vast majority of them residing in Africa (Global Statistics | HIV.Gov n.d.). In Tanzania, an estimated 93,000 children are living with HIV and $66 \%$ of them receiving ART (Global Statistics | HIV.Gov n.d.). In the absence of a cure, these children are set to receive ART throughout their lives and are thus vulnerable to HIV-mediated age-related illnesses which will not only impact their quality and length of life but will also overwhelm the already burdened healthcare systems of most resource-limited countries like Tanzania.

The accelerated aging experienced by HIV+ individuals also affects the immune system (Appay et al., 2007; Pathai et al., 2013), causing exhaustion of $B$ and $T$ immune cells, and inadequate response to vaccines (Pinti et al., 2016). Conditions associated with premature aging, including CVD risk, dyslipidemia and immune-aging have been poorly characterized in HIV+ African children (Gianesin et al., 2016), even though these illnesses may seriously threaten the future of these children. Hence, a need to identify and monitor for risk factors associated with CVD and immunoscenescence early on HIV+ children, even when they have suppression of viral replication and normal CD4 $\mathrm{T}$ cell counts. Therefore, this study sought to characterize premature aging in ART-treated HIV positive children focusing on lipid metabolic abnormalities as a risk factor for CVD, immune aging, and to understand their mutual relationship.

\section{MATERIALS AND METHODS}

\section{Study Population and Ethical Consideration}

One hundred and eleven age- and sex- matched HIV+ and HIVfemale and male children aged between 5 and 10 years were recruited into the study from June to December 2020 in Mbeya, Tanzania. HIV+ children $(n=64)$ were recruited from routine care and treatment pediatrics clinic at the Mbeya Referral Zonal Hospital (MZRH) in Mbeya, Tanzania. Healthy controls $(n=47)$ included children who had previously been treated for acute conditions at MZRH, children known by hospital staff and siblings/relatives $(n=2)$ of enrolled HIV+ children. This study was reviewed and approved by the Mbeya Medical Research and Ethics review Committee (SZEC-2439/R.A/V.1/61). Written Informed consent or assent was obtained from all parents/legal guardians and study volunteers and (when necessary) prior to any study related interview, clinical evaluation or sample acquisition.

\section{Clinical Assessments and Specimen Collection}

Interviews, clinical examination of the children and sample collection for laboratory assays were conducted by certified nurse and clinicians, and in accordance with the study approved protocol and national medical guidelines. A review of ART history, past and current medical diagnoses as well as demographics was carried out on HIV+ children using a questionnaire. Information on demographics and medical history was also collected from controls. Clinical examination included blood pressure and pulse rate measurements, height, weight and evaluation of participants' general health. For laboratory assays, peripheral blood was collected by venipuncture into SST tube (BD) for HIV rapid testing and clinical chemistry measurements, and into EDTA tubes (BD) for plasma HIV viral load and absolute CD4 T-cell quantification. Plasma obtained from the whole blood was used to quantify IgG antibody titers against measles and pertussis toxin and systemic IL6 levels by Enzyme linked Immunosorbent Assay (ELISA). Immediately upon collection, the samples were transported to the National Institute for Medical Research - Mbeya centre main laboratory.

\section{Clinical Chemistry (Triglycerides, HDL-c, LDL-c and Total Cholesterol)}

Clinical chemistry parameters in this study were measured by Cobas integra 400 (Roche). Normal reference ranges used were 
established by the National Institute for Medical Research Mbeya Medical Research Centre (NIMR-MMRC) for children living in Mbeya region, Tanzania (Supplementary Table 1). Lipid abnormalities/dyslipidemia were defined as having either or a combination of: Triglycerides $>2.18 \mathrm{~mm} / \mathrm{L}$ in males and $>2.88 \mathrm{~mm} / \mathrm{L}$ in females; HDL-c $<1.1 \mathrm{~mm} / \mathrm{L}$ in both males and females; LDL-c $>4.3 \mathrm{~mm} / \mathrm{L}$ in both males and females; total cholesterol $>5.5 \mathrm{~mm} / \mathrm{L}$ in both males and females.

\section{HIV Testing, ART Usage Information, CD4 Cell Counts and HIV Viral Load Quantification}

HIV status was determined by SD Bioline (Standard Diagnostics) and if positive confirmed by Unigold (Trinity Biotech). For HIV + children, CD4 counts were quantified by flow cytometry using either BD Facs Canto $^{\text {TM }}$ II (BD) or Facs Calibur ${ }^{\text {TM }}$ (BD). HIV plasma viral loads were quantified by Xpert HIV-1 Viral Load kit (Cepheid). Information on ART was obtained by interviewing guardians of HIV+ volunteers and from the hospital database. As per the current Tanzanian national guidelines (The United Republic of Tanzania, 2019), preferred first line ART regimen for children's with $<20 \mathrm{~kg}$ is a protease inhibitor (PI)-based regimen containing lopinavir and ritonavir (LPV/r) with nucleoside reverse transcriptase inhibitors (NRTI) combinations: abacavir and lamivudine (ABC/3TC). Integrase inhibitor, dolutegravir (DTG)-based regimen in combination with $\mathrm{ABC}$ and 3TC is recommended as the preferred $1^{\text {st }}$ line for children living with HIV weighing $\geq 20 \mathrm{~kg}$. Previously (The United Republic of Tanzania, 2017), the following drug combinations were recommended as $1^{\text {st }}$ line treatment for children:

- $\mathrm{ABC} / 3 \mathrm{TC}+\mathrm{LPV} / \mathrm{r}$ or zidovudine $(\mathrm{AZT}) / 3 \mathrm{TC}+\mathrm{LPV} / \mathrm{r}$

- Non-nucleoside reverse transcriptase inhibitor (NNRTI) regimen containing nevirapine (NVP) in combination with NRTIs, AZT/3TC

- NNRTI regimen containing efavirenz $(\mathrm{EFV})$ with one of the following NRTIs: AZT/3TC or ABC/3TC or tenofovir (TDF)/3TC

\section{ELISA for Human IL-6, IgG for Measles and Pertussis}

From sera samples, systemic human IL-6 levels were quantified by Human IL-6 ELISA kit (Invitrogen), anti-measles IgG titers were quantified by Measles IgG ELISA kit (Alpha Diagnostics) while anti-B. pertussis toxin IgG titers were quantified by Human anti-B. Pertussis Toxin/Toxoid (PTX) IgG ELISA kit (Alpha Diagnostics). For all three kits, manufacturers instruction were followed. Sunrise ELISA reader (Tecan) was used to measure the absorbance of the samples after the assays. Standards were used to determine the concentration of the respective analyte in relation to optical density determined by a plate reader. As per kit instructions, IgG titers were grouped as follows: For measles, below $8 \mathrm{U} / \mathrm{ml}$ was termed as negative, $8 \mathrm{U} / \mathrm{ml}$ to $12 \mathrm{U} / \mathrm{ml}$ was termed as equivocal and above $12 \mathrm{U} / \mathrm{ml}$ was regarded as a positive response. For pertussis, below $18 \mathrm{U} / \mathrm{ml}$ was termed as negative, $18 \mathrm{U} / \mathrm{ml}$ to $22 \mathrm{U} / \mathrm{ml}$ was termed as equivocal and $22 \mathrm{U} / \mathrm{ml}$ and above was regarded as a positive response. Cytokine and antibody concentrations below or above the measurable range were reported as the minimum or maximum measurable values respectively.

\section{Statistical Analysis}

Stata version 16 (StataCorp, USA) and GraphPad Prism software version 9 (GraphPad Software Inc, USA) were used for statistical analysis. Two tailed Mann-Whitney $U$ test was done to compare the quantities of lipids and plasma levels of human IL-6, Measles and Pertussis IgG titers when stratified by HIV status or by ARTbased regimens. Fisher's exact test was used to compare the proportion of different lipid clinical ranges between HIV+ and HIV- children. Spearman's rank correlation test was used to assess correlation between different variables. Multivariable poisson regression models were used to adjust for confounders, 95\% confidence interval and p-value was reported. Statistical significance was defined as $\mathrm{p}<0.05$.

\section{RESULTS}

\section{Description of the Study Participants}

A total of 111 children aged between 5 and 10 years were recruited into the study. Of these, 64 were HIV+ while 47 were HIV-. Groups were age- and sex-matched and had similar BMI and blood pressure measurements (Table 1). Within the HIV+ group, the median age at HIV diagnosis was 2.5 years. All of the HIV+ children were on ART with a median CD4 count of 887.5 cells $/ \mathrm{mm}^{3}$ and median plasma viral load copies of 39 copies/ml at the time of enrollment. At enrollment, $42 \%$ of children living with HIV (CLWH) were on protease inhibitor (PI) based regimen while 50\% were on Integrase inhibitor, dolutegravir (DTG)-based regimen and $8 \%$ were on NNRTI based regimen. Of note, 22\% (7/32) and 53\% (17/32) of $\mathrm{HIV}+$ children who are currently taking DTG-based regimen had previously been on PI- and NNRTI based ART regimens respectively. These were shifted to DTG therapy a median of 5.5 months ago. Table 1 details the demographic and clinical characteristics of study participants.

\section{Reduced HDL-c and Elevated Triglyceride Levels in HIV+ Children}

To determine the effect of HIV on lipid plasma levels, we stratified levels of cholesterol, triglycerides, HDL-c and LDL-c by HIV status. The prevalence of dyslipidaemia (as defined in the methods and Supplementary Table 1) was higher in HIV+ than of HIV - children [51.6\% (33/64) vs 27.7\% (13/47) respectively, $\mathrm{p}=0.019$, Table 2]. Specifically, $50 \%$ of CLWH had abnormally low concentrations of HDL-c while 27.7\% (13/47) of controls displayed such HDL-c abnormalities ( $\mathrm{p}=0.02$, Table 2). The prevalence of hypertriglyceridemia was $3.1 \%(2 / 64)$ vs $0 \%(0 / 47)$ for HIV+ and HIV- children respectively ( $p=0.507$, Table 2$)$. There were no individuals with abnormal levels of cholesterol and LDL-c in this study population (Table 2). We observed elevated triglycerides levels (median $\mathrm{mmol} / \mathrm{L}$ : 1.16 vs 0.81 , $\mathrm{p}<0.001$ ) but reduced HDL-c levels (median mmol/L: 1.1 vs 1.23, $\mathrm{p}<0.001)$ in HIV+ when compared to HIV- children 
TABLE 1 | Clinical parameters of the study participants.

\begin{tabular}{|c|c|c|c|}
\hline & HIV Negative $(n=47)$ & HIV Positive ( $n=64)$ & p-value ${ }^{a}$ \\
\hline Age (years) & $7(6-9)$ & $7.5(6-8.5)$ & 0.576 \\
\hline Gender (\% male) & 57.5 & 50 & $0.449^{*}$ \\
\hline Median age at HIV Diagnosis (years) & & $2.5(1.2-5.8)$ & \\
\hline CD4 counts (cells/ul) & & $887.5(620-1426)$ & \\
\hline HIV viremia (copies/mL) & & $39(0-39)$ & \\
\hline Duration of ART Usage (months) & & $57.5(13.6-76.6)$ & \\
\hline \%PI - Lopinavir/Ritonavir & & 42.2 & \\
\hline $\mathrm{BMI}\left(\mathrm{kg} / \mathrm{m}^{2}\right)$ & $15.5(14.8-16.1)$ & $15.6(15.1-16.6)$ & 0.134 \\
\hline Weight (kg) & $21.2(19-24.8)$ & $21.2(19-24.3)$ & 0.745 \\
\hline Height $(\mathrm{cm})$ & $118(112-124)$ & $116.7(110.1-122.2)$ & 0.137 \\
\hline Respiratory rate (beats/minute) & $22(22-24)$ & $22(21-24)$ & 0.835 \\
\hline Systolic Blood Pressure (mmHg) & $100(100-102)$ & $102(100-111)$ & 0.184 \\
\hline Diastolic Blood Pressure (mmHg) & $68(62-72)$ & $69(62-72)$ & 0.812 \\
\hline
\end{tabular}

All data are median (IQR) unless otherwise specified.

${ }^{a}$ Mann-Whitney U-test unless otherwise specified.

${ }^{*}$ Fishers exact test.

TABLE 2 | Prevalence of dyslipidaemia in HIV-and HIV+ children.

\begin{tabular}{lccc}
\hline Lipid Profile, $\mathbf{n}(\%)$ & HIV- ( $\mathbf{n = 4 7 )}$ & HIV+ ( $\mathbf{n}=\mathbf{6 4 )}$ & $\begin{array}{c}\mathbf{p} \text { value } \\
\text { (Fishers exact test) }\end{array}$ \\
\hline Overall dyslipidemia & $13(27.7 \%)$ & $33(51.6 \%)$ & 0.019 \\
Low HDL-C & $13(27.7 \%)$ & $32(50.0 \%)$ & 0.02 \\
Hypertriglyceridemia & $0(0 \%)$ & $2(3.1 \%)$ & 0.507
\end{tabular}

respectively (Figure 1A). Total cholesterol (median mmol/L: 3.4 vs 3.1, $\mathrm{p}=0.197$ ) and LDL-c (median $\mathrm{mmol} / \mathrm{L}: 2.0$ vs $1.8, \mathrm{p}=$ 0.264 ) plasma levels were not significantly higher in this study population of treated CLWH than in the controls (Figure 1A). Median triglycerides-HDL-c and LDL-c-HDL-c ratios were higher in HIV+ than HIV- children, 1.14 vs $0.64, \mathrm{p}<0.001$ and 1.5 vs $1.8, \mathrm{p}=0.005$, respectively (Figure 1B).

Similarly, Poisson regression model showed that HIV+ children had $48 \%$ relative risk (RR) increase in triglycerides when compared to HIV- children (CRR: 1.48, 95\% CI: 1.01 $2.15, \mathrm{p}=0.043$ ) and when adjusted for age, sex and BMI, we observed a $54 \% \mathrm{RR}$ increase in triglycerides among the HIV+ children (ARR: 1.54, 95\% CI: $1.31-1.82$, p < 0.001, Table 3). On the other hand, a $12 \% \mathrm{RR}$ in HDL-c reduction was found among the HIV+ when compared to HIV- children (CRR: 0.88, 95\%
A

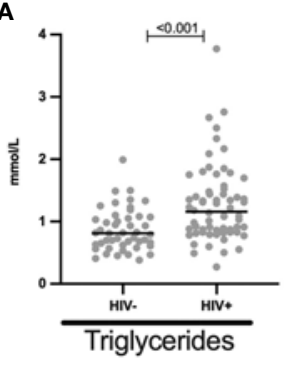

B

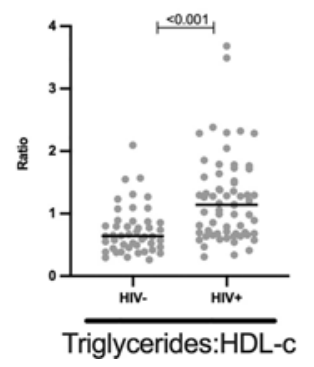

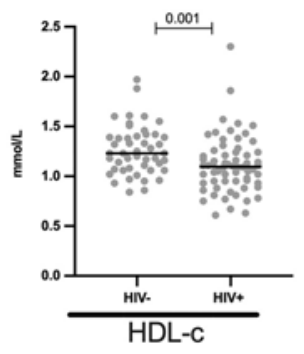

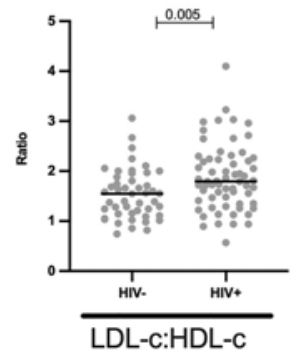

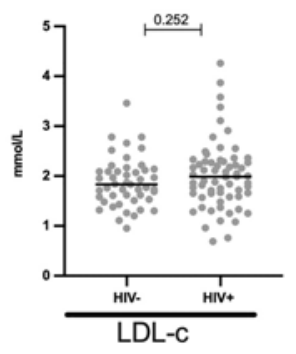

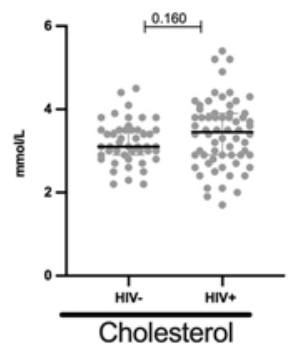

FIGURE 1 | Reduced HDL-c and increased triglycerides levels in HIV+ children: (A) Plasma quantity for triglycerides, HDL-C, LDL-C and cholesterol is shown in $\mathrm{mmol} / \mathrm{L}$ and stratified by HIV status. (B) Triglycerides-HDL-c ratio and LDL-C-HDL-c ratio is shown in HIV negative and HIV positive children. Each dot represents an individual volunteer, the median is indicated. Statistical analysis was performed using the Mann-Whitney U-test. 
CI:0.63 - 1.24, $\mathrm{p}=0.476$ ). After adjusting for confounders, there was an $11 \%$ RR for decreased HDL-c levels among the HIV+ (ARR: 0.89, 95\% CI: $0.82-0.96, p=0.003$, Table 3).

Together, these data indicate that HIV+ children have increased triglyceride and decreased HDL-c levels, and thus present with dyslipidaemia.

\section{Comparable IL-6 Plasma Levels in HIV+ and HIV- Children}

We next measured IL-6 plasma levels $(n=79)$ to determine the effect of HIV on systemic inflammation in ART experienced children (57 months median ART usage). We observed that the median IL-6 levels are comparable (median pg/ml: 3.2 vs 3.0, $\mathrm{p}=0.256$ ) between HIV + and HIV- children (Figure 2). Among HIV+ children, there was no correlation between ART duration and IL-6 plasma levels (Spearman $\mathrm{r}=0.006, \mathrm{p}=0.948$, Supplementary Figure 1).

\section{Reduced Measles and Pertussis IgG Plasma Levels in HIV+ Children}

To determine the effect HIV on childhood immunizations, we measured anti-measles $(n=80)$ and anti-pertussis $(n=77) \operatorname{IgG}$ titers in vaccinated children stratified by HIV status (Figure 3). According to the kits used, the results were grouped into either negative, equivocal or positive titer levels. The prevalence of positive measles vaccine-induced IgG titers was lower in HIV+ than HIV- children $[(14 \%(6 / 43)$ vs $43 \%(16 / 37) \mathrm{p}=0.005$, Table 4]. The proportion of positive IgG responses against pertussis toxin was $72.5 \%$ (29/40) in HIV+ and 86.5\% (32/37) HIV- children ( $p=0.165$, Table 4). In terms magnitude, HIV+ children, though on ART, had 2.8-fold reduced anti-measles IgG levels (Figure 3A) and 17.1-fold reduced anti-pertussis IgG levels (Figure 3B) when compared to HIV- children. Additionally, we observed an inverse correlation between age and anti-measles IgG titers in CLWH but not in HIV- children (Spearman $r=$ $-0.416, p=0.006$ for HIV+ and Spearman $r=-0.241, p=0.150$ for HIV-, Supplementary Figure 2). In samples with positive anti-measles and anti-pertussis IgG titers, median anti-measles

TABLE 3 | Multivariate Poisson regression modelling of association of dyslipidemia and HIV infection.

\begin{tabular}{|c|c|c|c|c|c|c|}
\hline Parameter & CRR & $95 \% \mathrm{Cl}$ & $p$-value & ARR & $95 \% \mathrm{Cl}$ & $\mathrm{p}$-value \\
\hline \multicolumn{7}{|l|}{ Triglycerides } \\
\hline \multicolumn{7}{|l|}{ HIV- } \\
\hline $\mathrm{HIV}+$ & 1.48 & $1.01-2.15$ & 0.043 & 1.54 & $1.31-1.82$ & $<0.001$ \\
\hline \multicolumn{7}{|l|}{ HDL-c } \\
\hline \multicolumn{7}{|l|}{ HIV- } \\
\hline HIV+ & 0.88 & $0.63-1.24$ & 0.476 & 0.89 & $0.82-0.96$ & 0.003 \\
\hline \multicolumn{7}{|l|}{ LDL-c } \\
\hline \multicolumn{7}{|l|}{ HIV- } \\
\hline HIV+ & 1.07 & $0.82-1.40$ & 0.619 & 1.08 & $0.96-1.20$ & 0.205 \\
\hline \multicolumn{7}{|l|}{ Cholesterol } \\
\hline $\mathrm{HIV}+$ & 1.06 & $0.86-1.31$ & 0.564 & 1.08 & $1.00-1.16$ & 0.054 \\
\hline
\end{tabular}

CRR, Crude risk ratio; ARR, Adjusted risk ratio; Cl, Confidence Interval; HDL-C, High density lipoprotein - cholesterol; LDL-c, Low density lipoprotein - cholesterol

The model was adjusted for age, sex and BMI.

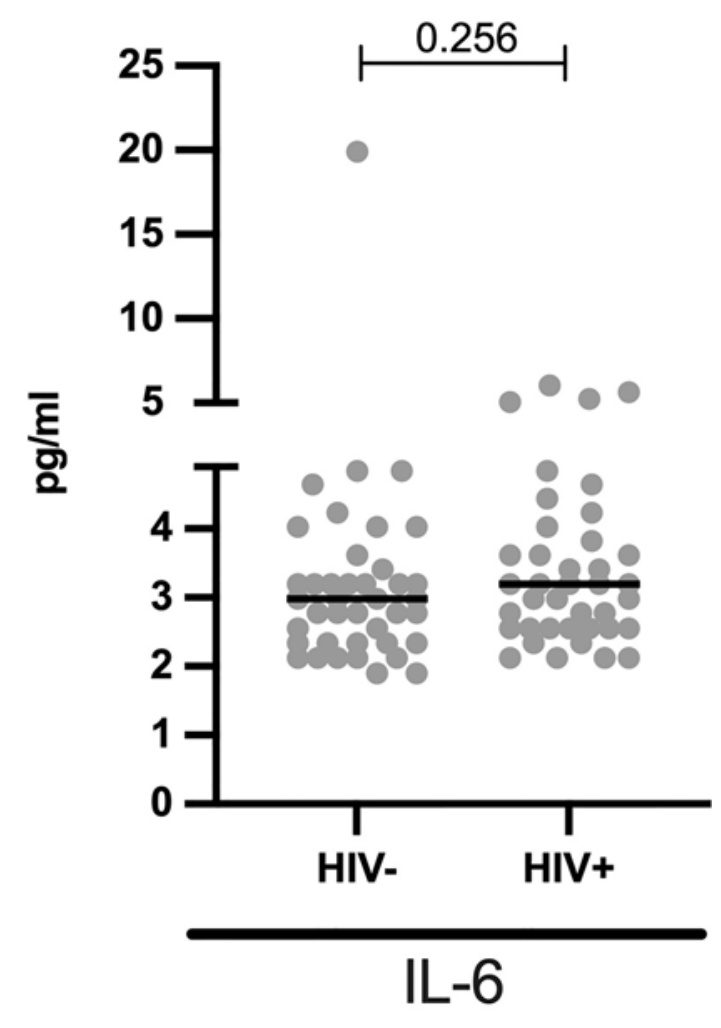

FIGURE 2 | Comparable systemic IL-6 levels in HIV- and HIV+ children: Concentrations of IL-6 (pg/ml) are stratified by HIV status. Each dot represents an individual volunteer, the median is indicated. Statistical analysis was performed using the Mann-Whitney U-test.

IgG titers were comparable between HIV+ and HIV- volunteers (median: $22.32 \mathrm{U} / \mathrm{ml}$ vs $24.86 \mathrm{U} / \mathrm{ml}$, p value $=0.858$, Figure $3 \mathrm{C}$ ) but an 8.6-fold reduction in median anti-pertussis IgG titers was observed in HIV+ children (median: $88.59 \mathrm{U} / \mathrm{ml}$ for HIV+ vs $775.2 \mathrm{U} / \mathrm{ml}$ for HIV-, $\mathrm{p}$ value $=0.013$, Figure 3D). This indicates a reduced humoral response to particularly anti-pertussis vaccine in these CLWH despite being on ART.

\section{Elevated Triglycerides and Cholesterol Levels in HIV+ on Lopinavir/Ritonavir Associated Inhibitors ART Regimen}

To determine the effect of PI, lopinavir/ritonavir on lipid profile of HIV+ children, we stratified data by HIV status and subsequently by lopinavir/ritonavir usage. We herein report that, among the $\mathrm{HIV}+$, triglyceride levels were slightly elevated in children on lopinavir/ritonavir (median mmol/L: 1.37) compared to those off lopinavir/ritonavir (median $\mathrm{mmol} /$ L:1.03, $p=0.032$, Figure 4). Similarly, cholesterol was increased in children on PI-based regimen when compared to those on DTG based treatment (median mmol/L: 3.6 vs 3.2, $\mathrm{p}=0.125$, Figure 4). Lopinavir/ritonavir did not affect plasma HDL-c and LDL-c levels in the studied population (Figure 4). Similarly, PI therapy was not associated with changes in IL-6, anti-measles and anti-pertussis IgG levels (Figure 4). 


\section{A}

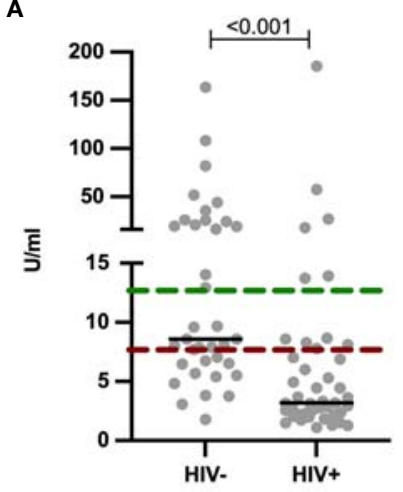

$\overline{\text { Anti-measles } \lg G}$

C

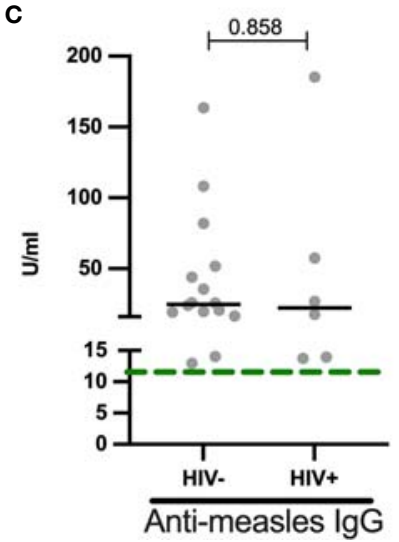

B

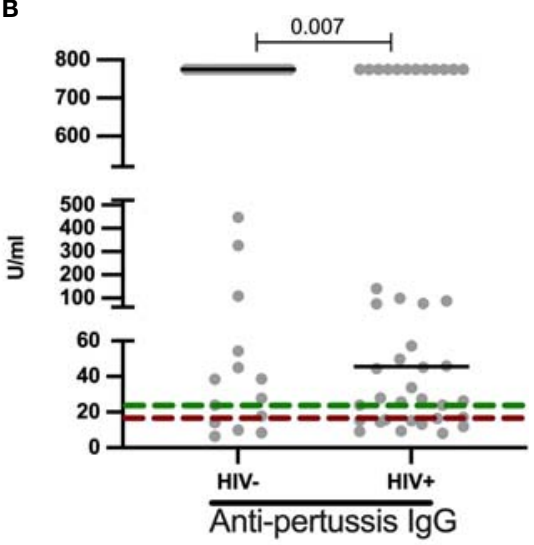

D

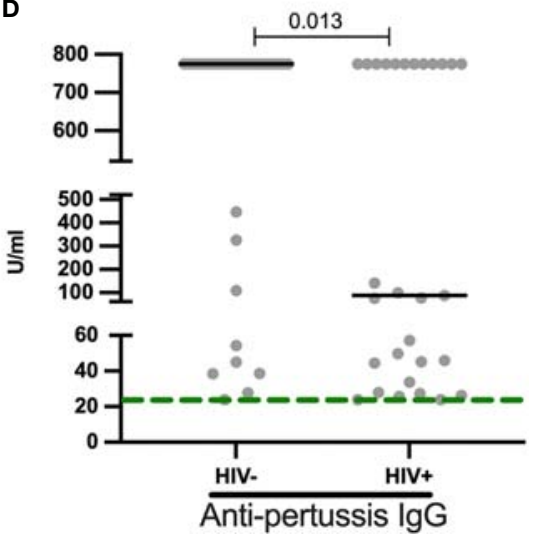

FIGURE 3 | Reduced anti-measles and anti-pertussis plasma lgG titers in HIV+ children: (A) Anti-measles (U/ml) and (B) anti-pertussis (U/ml) lgG titers are shown for HIV negative and HIV positive children. Individuals with positive (C) anti-measles (U/ml) and (D) anti-pertussis (U/ml) lgG titers are shown and stratified by HIV status. Titers above green dotted line are termed as positive, those between green and red dotted lines as equivocal and those below the red line as non-responses. Each dot represents an individual volunteer, the median is indicated. Statistical analysis was performed using the Mann-Whitney $U$-test.

TABLE 4 | Prevalence of anti-measles and anti-pertussis IgG responders in HIVand HIV+ children.

\begin{tabular}{lccc}
\hline Antibody, $\mathbf{n ~ ( \% ) ~}$ & HIV- & HIV+ & $\begin{array}{c}\text { p value } \\
\text { (Fishers exact test) }\end{array}$ \\
\hline Anti-Measles & 16 of $37(43.2 \%)$ & 6 of $43(14.0 \%)$ & 0.005 \\
Anti-Pertussis & 32 of 37 (86.5\%) & 29 of $40(72.5 \%)$ & 0.165 \\
\hline
\end{tabular}

\section{Association of Lipid Profiles With Immunity to Childhood Vaccines and Inflammation}

We found no significant correlation between lipid levels (HDL, triglycerides, LDL and cholesterol) and anti-measles and antipertussis titers (Figure 5). Similarly, no notable association was observed between each lipid and IL-6 (Figure 5).

\section{DISCUSSION}

People living with HIV with access to antiretroviral therapy (ART) are living longer, yet have significantly increased risk for age-related illnesses including cardiovascular disorders.
Premature aging and risk for cardiovascular disease (CVD) is understudied in children living with HIV, even though it is well known that subclinical CVD can begin in early childhood. Indeed, we observed that, $51.6 \%$ of CLWH had dyslipidemia compared to $27.7 \%$ of HIV- children. The prevalence of dyslipidemia was largely attributed to a higher proportion of abnormally low HDL-c and increased levels of triglycerides in $\mathrm{HIV}+$ children. These observations were further confirmed in a multivariate regression analysis, after adjusting for confounders. Our findings are comparable to prior studies that have analysed blood lipid disorders in adolescents and adults living with HIV living in similar settings (Ombeni and Kamuhabwa, 2016; Fiseha et al., 2021; Hamooya et al., 2021; Tilahun et al., 2021). A recent Zambian cross-sectional study found a high prevalence (63\%) of low HDL-c in ART experienced young adults living with HIV aged 18-24 years and that these young individuals were about three times more likely to have low HDL-c than older individuals (Hamooya et al., 2021). Also similar to our findings, the prevalence of low HDL-c and hypertriglyceridemia has also been observed in other studies with similar settings to be higher on ART experienced HIV+ than HIV- children 

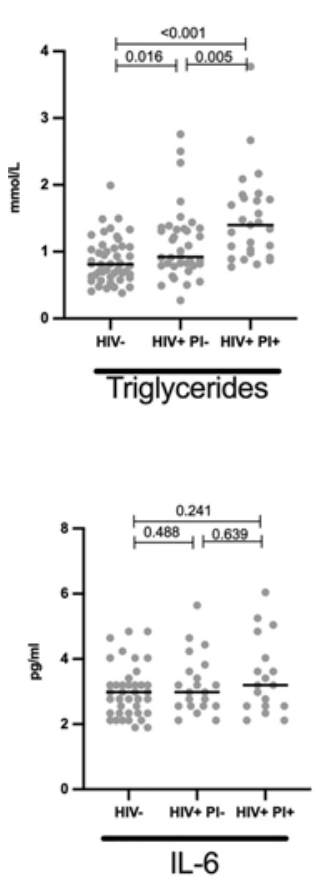
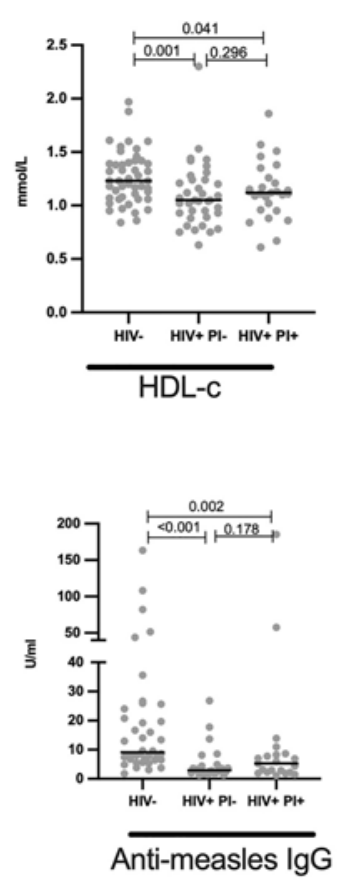
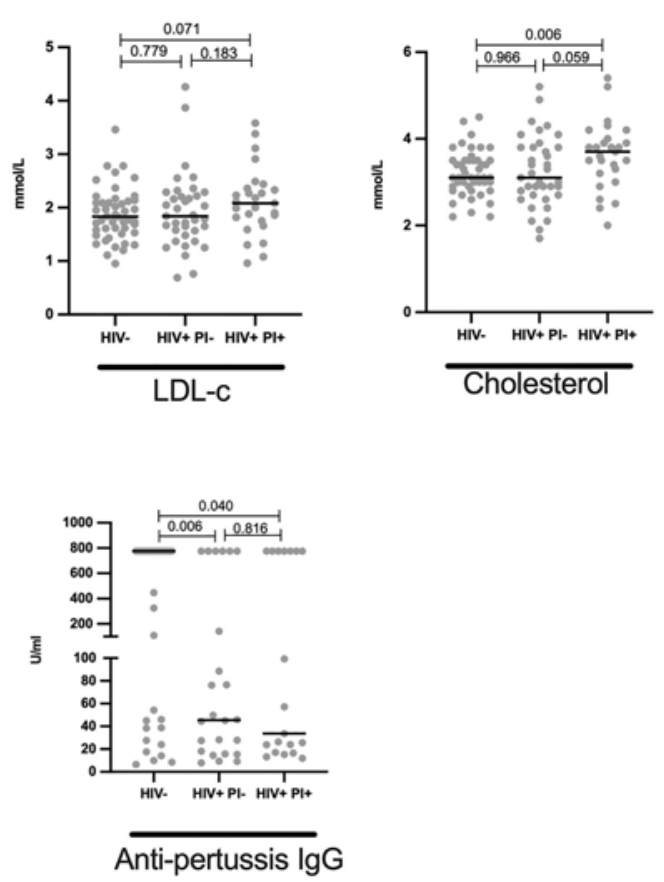

FIGURE 4 | Elevated levels of cholesterol and triglycerides in HIV+ children on protease inhibitor ART: Plasma quantity for triglycerides, HDL, LDL, cholesterol in mmol/L; IL-6 in pg/ml, anti-measles lgG and anti-pertussis in U/ml are shown and stratified by HIV status. Each dot represents an individual volunteer, the median is indicated Statistical analysis was performed using the Mann-Whitney U-test.

(Mandal et al., 2016; Dirajlal-Fargo et al., 2021). Dyslipidemia, particularly low HDL levels in treated HIV+ pediatrics has also been reported in Tanzanian and Ethiopian populations (Tadesse et al., 2019; Irira et al., 2020). However, to our knowledge, none of the earlier studies conducted in Africa have compared the level of dyslipidemia between age-matched HIV- and ART experienced HIV+ young children of less than 10 years.

While several studies have reported elevated systemic IL-6 levels in HIV+ individuals (Breen et al., 1990; Ullum et al., 1996; Shah et al., 2011; Gianesin et al., 2016), we here report similar IL-6 levels in HIV+ vs HIV-children. HIV associated factors such as duration of HIV infection, CD4 counts and HIV RNA plasma levels can affect IL-6 levels (Borges et al., 2015). We postulate that, there is no difference in IL-6 levels between the HIV+ vs HIV - children in our study because these children have been on ART for a median of 4.5 years, thus, have efficiently suppressed HIV viral replication and sufficiently restored their CD4 T -cell counts. Moreover, the children were HIV diagnosed in this test and treat era and so are likely to have had high nadir CD4 counts. In line with this, Borges et al. showed that IL-6 levels were increased in individuals with high HIV RNA levels with low nadir CD4 counts (Borges et al., 2015). Furthermore, Osuji and colleagues showed a 2.5 reduction in IL-6 levels just one year after ART initiation (Osuji et al., 2018). In similar recent studies, comparable levels of IL6 were observed between viral suppressing HIV+ children (from Africa and US) and their uninfected counterparts, further confirming our hypothesis (Wilkinson et al., 2018; Dirajlal-Fargo et al., 2020).
Ageing is characterized by increased dyslipidemia and blunted vaccine responses. To evaluate if such features also characterize HIV+ children, as a potential result of HIVinduced premature senescence, we measured antibody responses to childhood vaccination. In line with previous studies (Melvin and Mohan, 2003; Mutsaerts et al., 2018; Simani et al., 2019), we observed lower magnitude of antibody titers to measles and pertussis toxin in ART experienced CLWH. We also found a lower seropositivity prevalence to especially measles in CLWH on ART compared to HIV- children, suggesting inadequate measles vaccine-induced humoral response even in treated CLWH. Comparably, Pensieroso et al. observed the proportion of children from Italy with anti-measles seropositivity about 4 years post-vaccination to be $82 \%$ for HIV +ART+ children and 100\% for healthy controls (Pensieroso et al., 2009). Similarly, the prevalence of measles sero-protection (measured by neutralization assay) in CLWH on ART from the US was also found to be substantially lower than that of HIV exposed uninfected children (57\% vs 99\%) 9 years postvaccination (Siberry et al., 2015). Interestingly, within our cohort of HIV+ children, age was strongly associated with inadequate antibody responses to measles vaccine, further suggesting the accelerated aging processes even in ART experienced children.

Surprisingly, under half of healthy controls who reported to having received vaccination had protective IgG antibodies against measles. This is unlikely to be due to waning of protection with time, since anti-measles IgG antibodies are 

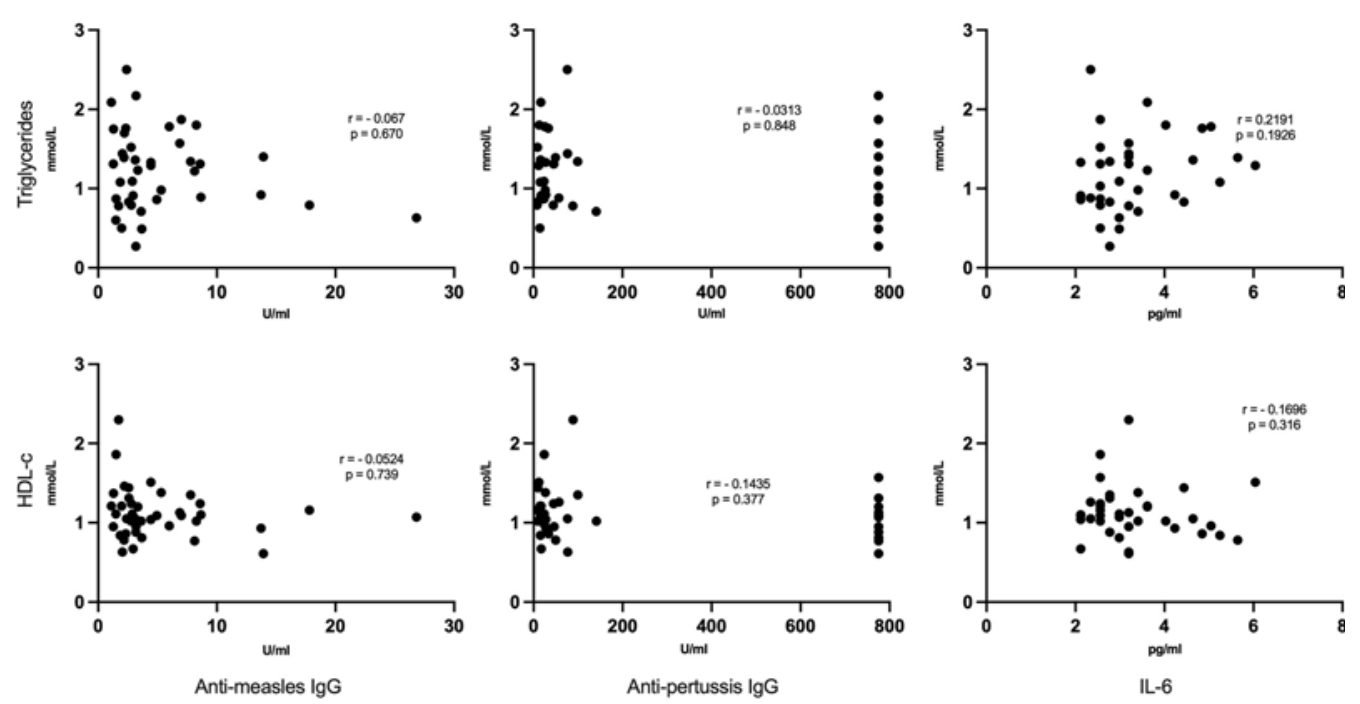

FIGURE 5 | No correlation between lipid levels and anti-measles, anti-pertussis plasma IgG titers or IL-6: Each dot represents respective lipid [triglycerides (upper panels) and HDL-c (lower panels)] and vaccine titers/L-6 serum levels. Statistical analysis was performed using the Spearman's rank correlation test.

known to persist for decades in healthy individuals (WHO n.d.). Since Measle-Rubella (MR) vaccine is usually given at a later stage in this region (9 and 18 months of age) compared to Diptheria-Tetanus and Pertussis (DTP) containing vaccine which is given 3 times before a child turns four months, it is plausible to think that some of the children reported to having received childhood immunization may have missed some vaccines including measles vaccine. Indeed, despite having one of the highest coverage rates of routine childhood immunization programme, over a quarter of children are not fully vaccinated in Tanzania (Tanzania, 2015; Vasudevan et al., 2020).

Previous studies (mostly done in HIV+ adults) have linked the use of protease (PI)-based ART with increased dyslipidaemia (Cunha, 2015; De Lima et al., 2018; Irira et al., 2020; Belete et al., 2021; Hamooya et al., 2021; Tilahun et al., 2021). HIV+ individuals on PI-based regimen are more likely to have higher concentrations of triglycerides than those who were on non-PI therapies (Kazooba et al., 2017; Belete et al., 2021). In this study, we similarly observed that CLWH receiving protease-based therapy (lopinavir boosted with ritonavir) had moderately higher triglyceride levels than CLWH who were on dolutegravir, an integrase-based regimen. These findings suggest that dyslipidaemia is not only influenced by HIV but is also strongly driven the exposure to PI-based regimens, highlighting the importance of switching to non-PI based therapies such as dolutegravir, which do not affect lipid composition (Quercia et al., 2015; Bagella et al., 2019).

To assess a possible link between the observed dyslipidemia and blunted immune response to vaccines in CLWH, Spearman's rank correlation test was used to analyse these parameters. We did not find any association between lipid levels and IgG antibody responses to measles and pertussis. To further elucidate the link between these two phenomena, future studies should further interrogate the relatedness between systemic metabolic disorders and immune dysregulation (such as T-cell senescence), since recent data indicate that lipid-containing tissues of HIV+ subjects are enriched with highly senescent CD8 ${ }^{+} \mathrm{T}$-cells (Godfrey et al., 2019), whose frequency is associated with CVD risk (Yu et al., 2016).

Our study was limited by its cross-sectional design; hence no causal inferences can be made. Moreover, lack of clinical CVD measurements limited correlating the observed CVD risk factors and immunoscenescence with clinical outcomes. The overall effect of ART on dyslipidemia and reduced vaccine-induced antibody responses could also not be elucidated in this study, since we unable enroll HIV+ART naïve comparator group due to an excellent rollout of ART in all diagnosed HIV+ Tanzanian children. Nonetheless, the main strength of our study was in matching the study groups to address confounding factors. The HIV+ and HIV- children were not only matched by age and sex, but also had similar BMI and blood pressure measurements (which may also influence the CVD outcomes). This allowed us to identify the extent of dyslipidemia and alteration of vaccineinduced humoral response in virologically controlling young CLWH in our setting.

The findings of this well-matched study complement those of earlier studies and strengthens the findings that dyslipidaemia driven by low HDL-c and hypertriglyceridemia is prevalent even in treated HIV+ African children with controlled viremia, suggesting that predisposition for CVD can begin much earlier in childhood, especially for persons living with HIV. High concentrations of triglycerides in CLWH were also associated with protease-based therapy, which could further increase CVD risk. Moreover, antimeasles and anti-pertussis toxin IgG titers were substantially reduced in vaccinated $\mathrm{HIV}+$ children, suggesting a compromised humoral response to measles and pertussis. Future studies should 
monitor the identified CVD risk factors and immune-senescence prospectively, in association with CVD clinical outcomes. Monitoring of these events will be a prerequisite for improving care of individuals who acquired HIV at an early age, which will: i) prevent the premature onset of non-communicable diseases, in particular cardiovascular and metabolic ones and; ii) prevent the accelerated senescence of their immune system and therefore the susceptibility to preventable diseases.

\section{DATA AVAILABILITY STATEMENT}

The raw data supporting the conclusions of this article will be made available by the authors, without undue reservation.

\section{ETHICS STATEMENT}

The studies involving human participants were reviewed and approved by Mbeya Medical Research and Ethics review Committee. Written informed consent to participate in this study was provided by the participants' legal guardian/next of kin.

\section{AUTHOR CONTRIBUTIONS}

All authors contributed to manuscript writing. WM, IM, FN, $\mathrm{CN}, \mathrm{LM}, \mathrm{PM}$, and MC conceived, designed and conducted the study. PM and MC supervised the project. WM, WO, PA, and MC participated in data curation and statistical analysis. All authors contributed to the article and approved the submitted version.

\section{REFERENCES}

Antiretroviral Therapy Cohort Collaboration and The Antiretroviral Therapy Cohort, Zwahlen, M., Harris, R., May, M., Hogg, R., Costagliola, D., et al. (2009). Mortality of HIV-Infected Patients Starting Potent Antiretroviral Therapy: Comparison With the General Population in Nine Industrialized Countries. Int. J. Epidemiol. 38 (6), 1624-1633. Oxford University Press. doi: 10.1093/ije/dyp306

Appay, V., Almeida, J., Sauce, D., Autran, B., and Papagno, L. (2007). Accelerated Immune Senescence and HIV-1 Infection. Exp. Gerontology 42 (5), 432-437. doi: 10.1016/j.exger.2006.12.003

Bagella, P., Squillace, N., Ricci, E., Gulminetti, R., De Socio, G. V., Taramasso, L., et al. (2019). Lipid Profile Improvement in Virologically Suppressed Hiv-1Infected Patients Switched to Dolutegravir/ Abacavir/Lamivudine: Data From the SCOLTA Project. Infection Drug Resistance 12, 1385-1391. Dove Medical Press Ltd. doi: 10.2147/IDR.S203813

Belete, A. M., Seifu, D., Menon, M., Amogne, W., Shewa, A., and Tefera, A. A. (2021). Serum Lipid Profiles of Patients Taking Efavirenz-Based Antiretroviral Regimen Compared to Ritonavir-Boosted Atazanavir With an Optimized Background at Zewditu Memorial Hospital, Addis Ababa, Ethiopia. HIV/AIDS - Res. Palliative Care 13, 217-227. Dove Medical Press Ltd. doi: 10.2147/HIV.S296170

Borges, ÁlvaroH., O’Connor, J. L., Phillips, A. N., Rönsholt, F. F., Pett, S., Vjecha, M. J., et al. (2015). Factors Associated With Plasma IL-6 Levels During HIV Infection. J. Infect. Dis. 212 (4), 585-595. Oxford University Press. doi: 10.1093/infdis/jiv123

Brady, M. T., Oleske, J. M., Williams, P. L., Elgie, C., Mofenson, L. M., Dankner, W. M., et al. (2010). Declines in Mortality Rates and Changes in Causes of

\section{FUNDING}

This work was supported by the University of Dar es Salaam (MCHAS-20022) and by Network Funds (PLAY HIGH and UAW project grants) from Ludwig Maximilians University of Munich (LMU)-Center for International Health (CIH), through DAAD/Exceed Program which is funded by the Ministry of Economic Efforts and Development of Germany (BMZ).

\section{ACKNOWLEDGMENTS}

We thank all the study volunteers and the research team members who participated in the recruitment of study participants, Veronica Mng'ong'o, Catherine Mwanamwene, and Editha Sanga. We also gratefully acknowledge the support of the laboratory personnel including Ernest Andrew and Stephen Msangi at the National Institute for Medical Research-Mbeya Medical Research Centre.

\section{SUPPLEMENTARY MATERIAL}

The Supplementary Material for this article can be found online at: https://www.frontiersin.org/articles/10.3389/fcimb.2021. 721747/full\#supplementary-material

Supplementary Figure 1 | No correlation between ART duration and IL-6 (pg/ml): Each dot represents an ART duration in months and IL-6 concentration pair for each individual volunteer. Statistical analysis was performed using the Spearman rank correlation test.

Supplementary Figure 2 | Negative correlation between anti-measles IgG titers and age in HIV+ children: Each dot represents a pair of anti-measles IgG titer and age in years for each individual volunteer. Statistical analysis was performed using the Spearman's rank correlation test.

Death in HIV-1-Infected Children During the HAART Era. J. Acquired Immune Deficiency Syndromes 53 (1), 86-94. NIH Public Access. doi: 10.1097/QAI.0b013e3181b9869f

Breen, E. C., Rezai, A. R., Nakajima, K., Beall, G. N., Mitsuyasu, R. T., Hirano, T., et al. (1990). Infection With HIV Is Associated With Elevated IL-6 Levels and Production. J. Immunol. 144 (2), 480-484. American Association of Immunologists.

Chiappini, E., Berti, E., Gianesin, K., Petrara, M. R., Galli, L., Giaquinto, C., et al. (2014). Pediatric Human Immunodeficiency Virus Infection and Cancer in the Highly Active Antiretroviral Treatment (HAART) Era. Cancer Lett. 347 (1), 38-45. Elsevier. doi: 10.1016/j.canlet.2014.02.002

Cunha, J. da (2015). Impact of Antiretroviral Therapy on Lipid Metabolism of Human Immunodeficiency Virus-Infected Patients: Old and New Drugs. World J. Virol. 4 (2), 56. Baishideng Publishing Group Inc. doi: 10.5501/ wjv.v4.i2.56

Daniels, S. R., Pratt, C. A., and Hayman, L. L. (2011). Reduction of Risk for Cardiovascular Disease in Children and Adolescents. Circulation 124 (15), 1673-1686. NIH Public Access. doi: 10.1161/CIRCULATIONAHA.110.016170

Deeks, S. G., and Phillips, A. N. (2009). HIV Infection, Antiretroviral Treatment, Ageing, and Non-AIDS Related Morbidity. BMJ 338, a3172. British Medical Journal Publishing Group. doi: 10.1136/bmj.a3172

De Lima, L. R. A., Petroski, E. L., Moreno, Y. M. F., Silva, D. A. S., de Moraes Santos Trindade, E. B., de Carvalho, A. P., et al. (2018). Dyslipidemia, Chronic Inflammation, and Subclinical Atherosclerosis in Children and Adolescents Infected With HIV: The PositHIVe Health Study. PloS One 13 (1), e0190785. Public Library of Science. doi: 10.1371/journal.pone.0190785 
Dirajlal-Fargo, S., Sattar, A., Yu, J., Albar, Z., Chaves, F. C., Riedl, K., et al. (2021). Lipidome Association With Vascular Disease and Inflammation in HIV+ Ugandan Children. AIDS Publish Ah. 35, 1615-1623. doi: 10.1097/ QAD. 0000000000002923

Dirajlal-Fargo, S., Shan, L., Sattar, A., Kulkarni, M., Bowman, E., Funderburg, N., et al. (2020). Micronutrients, Metabolic Complications, and Inflammation in Ugandan Children With HIV. J. Pediatr. Gastroenterol. Nutr. 70 (5), e100e105. NLM (Medline). doi: 10.1097/MPG.0000000000002630

Fiseha, T., Alemu, W., Dereje, H., Tamir, Z., and Gebreweld, A. (2021). Prevalence of Dyslipidaemia Among HIV-Infected Patients Receiving Combination Antiretroviral Therapy in North Shewa, Ethiopia. PloS One 16 (4), e0250328. Public Library of Science. doi: 10.1371/journal.pone.0250328

Gianesin, K., Noguera-Julian, A., Zanchetta, M., Del Bianco, P., Petrara, M. R., Freguja, R., et al. (2016). Premature Aging and Immune Senescence in HIVInfected Children. AIDS (London England) 30 (9), 1363-1373. Wolters Kluwer Health. doi: 10.1097/QAD.0000000000001093

Global Statistics | HIV.Gov N.D. Available at: https://www.hiv.gov/hiv-basics/ overview/data-and-trends/global-statistics (Accessed August 29, 2019).

Godfrey, C., Bremer, A., Alba, D., Apovian, C., Koethe, J. R., Koliwad, S., et al. (2019). Obesity and Fat Metabolism in Human Immunodeficiency Virus-Infected Individuals: Immunopathogenic Mechanisms and Clinical Implications. J. Infect. Dis. 220, 420-431. Oxford University Press. doi: 10.1093/infdis/jiz118

Guaraldi, G., Orlando, G., Zona, S., Menozzi, M., Carli, F., Garlassi, E., et al. (2011). Premature Age-Related Comorbidities Among HIV-Infected Persons Compared With the General Population. Clin. Infect. Dis. 53 (11), 1120-1126. Narnia. doi: 10.1093/cid/cir627

Guaraldi, G., and Palella, F. J. (2017). Clinical Implications of Aging With HIV Infection. AIDS 31, S129-S135. doi: 10.1097/QAD.0000000000001478

Hamooya, B. M., Musonda, P., Mutale, W., Masenga, S. K., Halwiindi, H., Mutengo, K. H., et al. (2021). Prevalence of Low High-Density Lipoprotein Among Young Adults Receiving Antiretroviral Therapy in Zambia: An Opportunity to Consider Non-Communicable Diseases in Resource-Limited Settings. PloS One 16, e0247004. Public Library of Science. doi: 10.1371/ journal.pone. 0247004

IeDEA Pediatric Working Group (2017). Taking a Critical Look at the UNAIDS Global Estimates on Paediatric and Adolescent HIV Survival and Death. J. Int. AIDS Soc. 20 (1), 21952. doi: 10.7448/IAS.20.1.21952

Irira, M. E., Philemon, R. N., Mmbaga, J. Y., Komba, V., Bartlett, J., Kinabo, G. D., et al. (2020). Dyslipidemia in HIV-Infected Children and Adolescents on Antiretroviral Therapy Receiving Care at Kilimanjaro Christian Medical Centre in Tanzania: A Cross-Sectional Study. Infect. Dis. 13, 1178633720948860. doi: 10.1177/1178633720948860

Jaquith, B. C., Harris, M. A., and Penprase, B. (2013). Cardiovascular Disease Risk in Children and Adolescents. J. Pediatr. Nurs. 28 (3), 258-266. doi: 10.1016/ j.pedn.2012.11.003

Kazooba, P., Kasamba, I., Nsubuga Mayanja, B., Lutaakome, J., Namakoola, I., Salome, T., et al. (2017). Cardiometabolic Risk Among HIV-Positive Ugandan Adults: Prevalence, Predictors and Effect of Long-Term Antiretroviral Therapy. Pan Afr. Med. J. 27, 40. African Field Epidemiology Network. doi: 10.11604/pamj.2017.27.40.9840

Maggi, P., Di Biagio, A., Rusconi, S., Cicalini, S., D’Abbraccio, M., d'Ettorre, G., et al. (2017). Cardiovascular Risk and Dyslipidemia Among Persons Living With HIV: A Review. BMC Infect. Dis. 17 (1), 551. BioMed Central. doi: 10.1186/s12879-017-2626-z

Mandal, A., Mukherjee, A., Lakshmy, R., Kabra, S. K., and Lodha, R. (2016). Dyslipidemia in HIV Infected Children Receiving Highly Active Antiretroviral Therapy. Indian J. Pediatr. 83 (3), 226-231. Springer India. doi: 10.1007/ s12098-015-1859-3

McDonald, C. L., and Kaltman, J. R. (2009). Cardiovascular Disease in Adult and Pediatric HIV/AIDS. J. Am. Coll. Cardiol. 54 (13), 1185-1188. NIH Public Access. doi: 10.1016/j.jacc.2009.05.055

Melvin, A. J., and Mohan, K. M. (2003). Response to Immunization With Measles, Tetanus, and Haemophilus Influenzae Type B Vaccines in Children Who Have Human Immunodeficiency Virus Type 1 Infection and Are Treated With Highly Active Antiretroviral Therapy. Pediatrics 111 (6 Pt 1), e641-e644. Pediatrics. doi: 10.1542/peds.111.6.e641

Modrich, L., Scherzer, R., Zolopa, A., Rimland, D., Lewis, C. E., Bacchetti, P., et al. (2010. Association of HIV Infection, Demographic and Cardiovascular Risk
Factors With All-Cause Mortality in the Recent HAART Era. J. Acquired Immune Deficiency Syndromes 53 (1), 102. NIH Public Access. doi: 10.1097/ QAI.0b013e3181b79d22

Mutsaerts, E. A.M.L., Nunes, M. C., van Rijswijk, M. N., Klipstein-Grobusch, K., Grobbee, D. E., Madhi, S. A., et al. (2018). Safety and Immunogenicity of Measles Vaccination in HIV-Infected and HIV-Exposed Uninfected Children: A Systematic Review and Meta-Analysis. EClinicalMedicine 1, 28-42. Elsevier. doi: 10.1016/j.eclinm.2018.06.002

Nduka, C., Sarki, A., Uthman, O., and Stranges, S. (2015). Impact of Antiretroviral Therapy on Serum Lipoprotein Levels and Dyslipidemias: A Systematic Review and Meta-Analysis. Int. J. Cardiol. 199, 307-318. Elsevier Ireland Ltd. doi: 10.1016/j.ijcard.2015.07.052

Ombeni, W., and Kamuhabwa, A. R. (2016). Lipid Profile in HIV-Infected Patients Using First-Line Antiretroviral Drugs. J. Int. Assoc. Providers AIDS Care 15 (2), 164-171. SAGE Publications Inc. doi: 10.1177/2325957415614642

Osuji, F. N., Onyenekwe, C. C., Ahaneku, J. E., and Ukibe, N. R. (2018). The Effects of Highly Active Antiretroviral Therapy on the Serum Levels of ProInflammatory and Anti-Inflammatory Cytokines in HIV Infected Subjects 11 Medical and Health Sciences 1103 Clinical Sciences 11 Medical and Health Sciences 1107 Immunology. J. Biomed. Sci. 25 (1), 1-8. BioMed Central Ltd. doi: 10.1186/s12929-018-0490-9

Paisible, A. L., Chang, C. C. H., So-Armah, K. A., Butt, A. A., Leaf, D. A., Budoff, M., et al. (2015). HIV Infection, Cardiovascular Disease Risk Factor Profile, and Risk for Acute Myocardial Infarction. J. Acquired Immune Deficiency Syndromes 68 (2), 209-216. Lippincott Williams and Wilkins. doi: 10.1097/ QAI.0000000000000419

Pathai, S., Lawn, S. D., Gilbert, C. E., McGuinness, D., McGlynn, L., Weiss, H. A., et al. (2013). Accelerated Biological Ageing in HIV-Infected Individuals in South Africa: A Case-Control Study. AIDS (London England) 27 (15), 23752384. Wolters Kluwer Health. doi: 10.1097/QAD.0b013e328363bf7f

Pensieroso, S., Cagigi, A., Palma, P., Nilsson, A., Capponi, C., Freda, E., et al. (2009). Timing of HAART Defines the Integrity of Memory B Cells and the Longevity of Humoral Responses in HIV-1 Vertically-Infected Children. Proc. Natl. Acad. Sci. U.S.A. 106 (19), 7939-7944. National Academy of Sciences. doi: 10.1073/pnas.0901702106

Pinti, M., Appay, V., Campisi, J., Frasca, D., Fülöp, T., Sauce, D., et al. (2016). Aging of the Immune System: Focus on Inflammation and Vaccination. Eur. J. Immunol. 46 (10), 2286-2301. NIH Public Access. doi: 10.1002/ eji.201546178

Quercia, R., Roberts, J., Martin-Carpenter, L., and Zala, C. (2015). Comparative Changes of Lipid Levels in Treatment-Naive, HIV-1-Infected Adults Treated With Dolutegravir vs. Efavirenz, Raltegravir, and Ritonavir-Boosted Darunavir-Based Regimens Over 48 Weeks. Clin. Drug Invest. 35 (3), 211219. Springer International Publishing. doi: 10.1007/s40261-014-0266-2

Rahmanian, S., Wewers, M. E., Koletar, S., Reynolds, N., Ferketich, A., and Diaz, P. (2011). "Cigarette Smoking in the Hiv-Infected Population," in Proceedings of the American Thoracic Society (American Thoracic Society), pp. 313-319.

Shah, A., Verma, A. S., Patel, K. H., Noel, R., Rivera-Amill, V., Silverstein, P. S., et al. (2011). HIV-1 Gp120 Induces Expression of IL-6 Through a Nuclear Factor-Kappa B-Dependent Mechanism: Suppression by Gp120 Specific Small Interfering RNA. PloS One 6 (6), e21261. Public Library of Science. doi: 10.1371/journal.pone.0021261

Siberry, G. K., Patel, K., Bellini, W. J., Karalius, B., Purswani, M. U., Burchett, S. K., et al. (2015). Immunity to Measles, Mumps, and Rubella in US Children With Perinatal HIV Infection or Perinatal HIV Exposure Without Infection. Clin. Infect. Dis. 61 (6), 988-995. Oxford University Press. doi: 10.1093/cid/ civ440

Simani, O. E., Izu, A., Nunes, M. C., Violari, A., Cotton, M. F., Van Niekerk, N., et al. (2019). Effect of HIV Exposure and Timing of Antiretroviral Therapy Initiation on Immune Memory Responses to Diphtheria, Tetanus, Whole Cell Pertussis and Hepatitis B Vaccines. Expert Rev. Vaccines 18 (1), 95-104. Taylor and Francis Ltd. doi: 10.1080/14760584.2019.1547195

Syed, S. S., Balluz, R. S., Kabagambe, E. K., Meyer, W. A., Lukas, S., Wilson, C. M., et al. (2013). Assessment of Biomarkers of Cardiovascular Risk Among HIV Type 1-Infected Adolescents: Role of Soluble Vascular Cell Adhesion Molecule as an Early Indicator of Endothelial Inflammation. AIDS Res. Hum. Retroviruses 29 (3), 493-500. Mary Ann Liebert, Inc. doi: 10.1089/ aid.2012.0086 
Tadesse, B. T., Foster, B. A., Chala, A., Chaka, T. E., Bizuayehu, T., Ayalew, F., et al. (2019). HIV and CART-Associated Dyslipidemia Among HIV-Infected Children. J. Clin. Med. 8 (4), 430. doi: 10.3390/jcm8040430

Tanzania, United Republic of (2015). Tanzania Demographic and Health Survey and Malaria Indicator Survey.

The United Republic of Tanzania (2017). NATIONAL GUIDELINES FOR THE MANAGEMENT OF HIV AND AIDS.

The United Republic of Tanzania (2019). NATIONAL GUIDELINES FOR THE MANAGEMENT OF HIV AND AIDS.

Tien, P. C., Schneider, M. F., Cox, C., Karim, R., Cohen, M., Sharma, A., et al. (2012). Association of HIV Infection With Incident Diabetes Mellitus: Impact of Using Hemoglobin $\mathrm{A} 1 \mathrm{C}$ as a Criterion for Diabetes. J. Acquired Immune Deficiency Syndromes 61 (3), 334-340. NIH Public Access. doi: 10.1097/ QAI.0b013e31826bfc32

Tilahun, H., Masyuko, S. J., Mogaka, J. N., Temu, T., Kinuthia, J., Osoti, A. O., et al. (2021). Prevalence and Correlates of Dyslipidemia in HIV Positive and Negative Adults in Western Kenya: A Cross-Sectional Study. Medicine 100 (10), e24800. NLM (Medline). doi: 10.1097/MD.0000000000024800

Ullum, H., Diamant, M., Victor, J., Gøtzsche, P. C., Bendtzen, K., Skinhøj, P., et al. (1996). Increased Circulating Levels of Interleukin-6 in HIV-Seropositive Subjects. JAIDS J. Acquired Immune Deficiency Syndromes 13 (1), 93. doi: 10.1097/00042560-199609000-00013

Vasudevan, L., Baumgartner, J. N., Moses, S., et al. (2020). Parental Concerns and Uptake of Childhood Vaccines in Rural Tanzania - a Mixed Methods Study. BMC Public Health 20 (1), 1-11. BioMed Central Ltd. doi: 10.1186/s12889020-09598-1
WHO. Weekly Epidemiological Record Relevé Épidémiologique Hebdomadaire.

Wilkinson, J. D., Williams, P. L., Yu, W., Colan, S. D., Mendez, A., Zachariah, J. P. V., et al. (2018). Cardiac and Inflammatory Biomarkers in Perinatally HIVInfected and HIV-Exposed Uninfected Children. AIDS 32 (10), 1267-1277. Lippincott Williams and Wilkins. doi: 10.1097/QAD.0000000000001810

Yu, H. T., Park, S., Shin, E. C., and Lee, W. W. (2016). T Cell Senescence and Cardiovascular Diseases. Clin. Exp. Med. 16, 257-263. Springer-Verlag Italia s.r.l. doi: $10.1007 /$ s10238-015-0376-Z

Conflict of Interest: The authors declare that the research was conducted in the absence of any commercial or financial relationships that could be construed as a potential conflict of interest.

Publisher's Note: All claims expressed in this article are solely those of the authors and do not necessarily represent those of their affiliated organizations, or those of the publisher, the editors and the reviewers. Any product that may be evaluated in this article, or claim that may be made by its manufacturer, is not guaranteed or endorsed by the publisher.

Copyright (C) 2021 Mbuya, Mwakyula, Olomi, Agrea, Nicoli, Ngatunga, Mujwahuzi, Mwanyika and Chachage. This is an open-access article distributed under the terms of the Creative Commons Attribution License (CC BY). The use, distribution or reproduction in other forums is permitted, provided the original author(s) and the copyright owner(s) are credited and that the original publication in this journal is cited, in accordance with accepted academic practice. No use, distribution or reproduction is permitted which does not comply with these terms. 